\title{
Long Range Scattering and Modified Wave Operators for the Wave-Schrödinger System II*
}

\author{
J. Ginibre \\ Laboratoire de Physique Théorique ${ }^{\dagger}$ \\ Université de Paris XI, Bâtiment 210, F-91405 ORSAY Cedex, France \\ G. Velo \\ Dipartimento di Fisica, Università di Bologna \\ and INFN, Sezione di Bologna, Italy
}

\begin{abstract}
We continue the study of scattering theory for the system consisting of a Schrödinger equation and a wave equation with a Yukawa type coupling in space dimension 3 . In a previous paper, we proved the existence of modified wave operators for that system with no size restriction on the data and we determined the asymptotic behaviour in time of solutions in the range of the wave operators, under a support condition on the asymptotic state required by the different propagation properties of the wave and Schrödinger equations. Here we eliminate that condition by using an improved asymptotic form for the solutions.
\end{abstract}

AMS Classification : Primary 35P25. Secondary 35B40, 35Q40, 81U99.

Key words : Long range scattering, modified wave operators, Wave-Schrödinger system.

LPT Orsay 03-07

February 2003

\footnotetext{
${ }^{*}$ Work supported in part by NATO Collaborative Linkage Grant 979341
}

${ }^{\dagger}$ Unité Mixte de Recherche (CNRS) UMR 8627 


\section{Introduction}

This paper is a sequel to a previous paper with the same title ([1], hereafter referred to as I) where we studied the theory of scattering and proved the existence of modified wave operators for the Wave-Schrödinger (WS) system

$$
\left\{\begin{array}{l}
i \partial_{t} u=-(1 / 2) \Delta u-A u \\
\square A=|u|^{2}
\end{array}\right.
$$

where $u$ and $A$ are respectively a complex valued and a real valued function defined

in space time $\mathbb{R}^{3+1}$. We refer to the introduction of I for general background and references and we give here only a general overview of the problem.

The main result of I was the construction of modified wave operators for the WS system, with no size restriction on the solutions. That construction basically consists in solving the Cauchy problem for the WS system with infinite initial time, namely in constructing solutions with prescribed asymptotic behaviour at infinity in time. That asymptotic behaviour is imposed in the form of suitable approximate solutions of the WS system. One then looks for exact solutions, the difference of which with the given approximate ones tends to zero at infinity in time in a suitable sense, more precisely in suitable norms. The approximate solutions are obtained as low order iterates in an iterative resolution scheme of the WS system. In I we used second order iterates. They are parametrized by data $\left(u_{+}, A_{+}, \dot{A}_{+}\right)$which play the role of (actually are in simpler cases) initial data at time zero. Those data constitute the asymptotic state for the actual solution.

An inherent difficulty of the WS system is the difference of propagation properties of the wave equation and of the Schrödinger equation. Because of that difficulty, we had to impose in I a support condition on the Fourier transform $F u_{+}$of the Schrödinger asymptotic state $u_{+}$, saying in effect that $F u_{+}$vanishes in a neighborhood of the unit sphere, so that $u_{+}$generates a solution of the free Schrödinger equation which is asymptotically small in a neighborhood of the light cone. Such a support condition is unpleasant because it cannot be satisfied on a dense subspace of any reasonable space where one hopes to solve the problem, typically with $u$ in $F H^{k}$ for $k>1 / 2\left(H^{k}\right.$ is the standard $L^{2}$ based Sobolev space).

The theory of scattering and the existence of modified wave operators can also be studied for various equations and systems including the WS system by a method simpler than that of I, proposed earlier by Ozawa [5]. Contrary to that of I, that 
method is restricted to the case of small data and small solutions. It has been applied to various systems, in particular to the Klein-Gordon-Schrödinger (KGS) system in dimension 2 , which is fairly similar to the WS system in dimension 3 from the point of view of scattering [6]. Similar propagation difficulties also appear for that system, thereby again requiring a support condition on $F u_{+}$in the treatment given in [6].

A progress on that problem was made recently by Shimomura [7] [8] who was able to remove the previous support condition in the construction of the modified wave operators by the Ozawa method in the case of the KGS system in dimension 2 [7] and of the WS system in dimension 3 [8]. The key of that progress consists in using an improved asymptotic form for the Schrödinger function, obtained by adding a term depending on $\left(A_{+}, \dot{A}_{+}\right)$which partly cancels the contribution of the asymptotic field for $A$ in the Schrödinger equation.

Although the method used in I is more complicated than the Ozawa method (so as to accomodate arbitrarily large data and solutions), it turns out that the improved asymptotic form of $u$ used in [8] can be transposed into the framework of the method of I, thereby allowing to remove the support condition on $F u_{+}$assumed in I, at least in a restricted interval of values of the parameters defining the regularity of the solutions. The purpose of the present paper is to implement that improvement, namely to rederive the main results of I without assuming the support condition used in I, by using the improved asymptotic form of the solution inspired by that of [8].

In the remaining part of this introduction, we shall briefly review the method used in I in the modified form used in the present paper. We refer to Section 2 of I for a more detailed exposition. The main result of this paper will be stated in semi heuristic terms at the end of this introduction. The first step in that method consists in eliminating the wave equation (1.2) by solving it for $A$ and substituting the result into the Schrödinger equation, which then becomes both non linear and non local in time. One then parametrizes the Schrödinger function $u$ in terms of an amplitude $w$ and a phase $\varphi$ and one replaces the Schrödinger equation by an auxiliary system consisting of a transport equation for the amplitude and a Hamilton-Jacobi equation for the phase. One solves the Cauchy problem at infinity, namely with prescribed asymptotic behaviour, for the auxiliary system, and one finally reconstructs the solution of the original WS system from that of the auxiliary system. We now proceed to the technical details. We restrict our attention to positive time, actually 
to $t \geq 1$.

We first eliminate the wave equation. We define

$$
\omega=(-\Delta)^{1 / 2} \quad, \quad K(t)=\omega^{-1} \sin \omega t \quad, \quad \dot{K}(t)=\cos \omega t
$$

and we replace $(1.2)$ by

$$
A=A_{0}+A_{1}\left(|u|^{2}\right)
$$

where

$$
\begin{gathered}
A_{0}=\dot{K}(t) A_{+}+K(t) \dot{A}_{+}, \\
A_{1}\left(|u|^{2}\right)=-\int_{t}^{\infty} d t^{\prime} K\left(t-t^{\prime}\right)\left|u\left(t^{\prime}\right)\right|^{2} .
\end{gathered}
$$

Here $A_{0}$ is a solution of the free wave equation with initial data $\left(A_{+}, \dot{A}_{+}\right)$at time $t=0$. The pair $\left(A_{+}, \dot{A}_{+}\right)$is the asymptotic state for $A$.

We next perform the change of variables mentioned above on $u$. The unitary group

$$
U(t)=\exp (i(t / 2) \Delta)
$$

which solves the free Schrödinger equation can be written as

$$
U(t)=M(t) D(t) F M(t)
$$

where $M(t)$ is the operator of multiplication by the function

$$
M(t)=\exp \left(i x^{2} / 2 t\right)
$$

$F$ is the Fourier transform and $D(t)$ is the dilation operator

$$
D(t)=(i t)^{-n / 2} D_{0}(t)
$$

where

$$
\left(D_{0}(t) f\right)(x)=f(x / t) .
$$

We parametrize $u$ in terms of an amplitude $w$ and of a real phase $\varphi$ as

$$
u(t)=M(t) D(t) \exp [-i \varphi(t)] w(t) .
$$

Substituting (1.11) into (1.1) yields an evolution equation for $(w, \varphi)$, namely

$$
\left\{i \partial_{t}+\left(2 t^{2}\right)^{-1} \Delta-i\left(2 t^{2}\right)^{-1}(2 \nabla \varphi \cdot \nabla+\Delta \varphi)+t^{-1} B+\partial_{t} \varphi-\left(2 t^{2}\right)^{-1}|\nabla \varphi|^{2}\right\} w=0
$$


where we have expressed $A$ in terms of a new function $B$ by

$$
A=t^{-1} D_{0} B \text {. }
$$

Corresponding to the decomposition (1.3) of $A$, we decompose

$$
B=B_{0}+B_{1}(w, w)
$$

where $A_{0}=t^{-1} D_{0} B_{0}$ and $A_{1}=t^{-1} D_{0} B_{1}$. One computes easily

$$
B_{1}\left(w_{1}, w_{2}\right)=\int_{1}^{\infty} d \nu \nu^{-3} \omega^{-1} \sin ((\nu-1) \omega) D_{0}(\nu)\left(\operatorname{Re} \bar{w}_{1} w_{2}\right)(\nu t) .
$$

At this point, we have only one evolution equation (1.12) for two functions $(w, \varphi)$. We arbitrarily impose a second equation, namely a Hamilton-Jacobi (or eikonal) equation for the phase $\varphi$, thereby splitting the equation (1.12) into a system of two equations, the other one of which being a transport type equation for the amplitude $w$. For that purpose, we split $B_{0}$ and $B_{1}$ into a long range and short range parts as follows. Let $\chi \in \mathcal{C}^{\infty}\left(\mathbb{R}^{3}, \mathbb{R}\right), 0 \leq \chi \leq 1, \chi(\xi)=1$ for $|\xi| \leq 1, \chi(\xi)=0$ for $|\xi| \geq 2$ and let $0<\beta_{0}, \beta<1$. We define

$$
B_{0}=B_{0 L}+B_{0 S} \quad, \quad B_{1}=B_{L}+B_{S}
$$

where

$$
\left\{\begin{array}{l}
F B_{0 L}(t, \xi)=\chi\left(\xi t^{-\beta_{0}}\right) F B_{0}(t, \xi), \\
F B_{L}(t, \xi)=\chi\left(\xi t^{-\beta}\right) F B_{1}(t, \xi) .
\end{array}\right.
$$

The splitting (1.16) (1.17) differs from that made in $I$ in two respects. First and more important is the fact that we perform that splitting both on $B_{0}$ and on $B_{1}$, whereas in I it was done only on $B_{1}$. Second, we use here a smooth cut-off $\chi$ instead of a sharp one. The smooth cut-off is actually needed only for $B_{0}$. For $\beta=\beta_{0}$, the splitting is the same for $B_{0}$ and $B_{1}$ and can therefore be performed on $B$ without any reference to the asymptotic state $\left(A_{+}, \dot{A}_{+}\right)$. The parameters $\beta_{0}$ and $\beta$ will have to satisfy various conditions which will appear later, all of them compatible with $\beta=\beta_{0}=1 / 3$.

We split the equation (1.12) into the following system of two equations.

$$
\left\{\begin{array}{l}
\partial_{t} w=i\left(2 t^{2}\right)^{-1} \Delta w+t^{-2} Q(\nabla \varphi, w)+i t^{-1}\left(B_{0 S}+B_{S}(w, w)\right) w \\
\partial_{t} \varphi=\left(2 t^{2}\right)^{-1}|\nabla \varphi|^{2}-t^{-1} B_{0 L}-t^{-1} B_{L}(w, w)
\end{array}\right.
$$


where we have defined

$$
Q(s, w)=s \cdot \nabla w+(1 / 2)(\nabla \cdot s) w
$$

for any vector field $s$. The first equation of (1.18) is the transport type equation for the amplitude $w$, while the second one is the Hamilton-Jacobi type equation for the phase $\varphi$. Since the right-hand sides of (1.18) contain $\varphi$ only through its gradient, we can obtain from (1.18) a closed system for $w$ and $s=\nabla \varphi$ by taking the gradient of the second equation, namely

$$
\left\{\begin{array}{l}
\partial_{t} w=i\left(2 t^{2}\right)^{-1} \Delta w+t^{-2} Q(s, w)+i t^{-1}\left(B_{0 S}+B_{S}(w, w)\right) w \\
\partial_{t} s=t^{-2} s \cdot \nabla s-t^{-1} \nabla B_{0 L}-t^{-1} \nabla B_{L}(w, w) .
\end{array}\right.
$$

Once the system (1.20) is solved for $(w, s)$, one recovers $\varphi$ easily by integrating the second equation of (1.18) over time. The system (1.20) will be referred to as the auxiliary system.

The construction of the modified wave operators follows the same pattern as in I. The first task is to construct solutions of the auxiliary system (1.20) with suitably prescribed asymptotic behaviour at infinity, and in particular with $w(t)$ tending to a limit $w_{+}=F u_{+}$as $t \rightarrow \infty$. That asymptotic behaviour is imposed in the form of a suitably chosen pair $(W, \phi)$ and therefore $(W, S)$ with $S=\nabla \phi$ with $W(t)$ tending to $w_{+}$as $t \rightarrow \infty$. For fixed $(W, S)$, we make a change of variables in the system (1.18) from $(w, \varphi)$ to $(q, \psi)$ defined by

$$
(q, \psi)=(w, \varphi)-(W, \phi)
$$

or equivalently a change of variables in the system $(1.20)$ from $(w, s)$ to $(q, \sigma)$ defined by

$$
(q, \sigma)=(w, s)-(W, S)
$$

and instead of looking for a solution $(w, s)$ of the system (1.20) with $(w, s)$ behaving asymptotically as $(W, S)$, we look for a solution $(q, \sigma)$ of the transformed system with $(q, \sigma)$ (and also $\psi$ ) tending to zero as $t \rightarrow \infty$. Performing the change of variables (1.22) in the auxiliary system (1.20) yields the following modified auxiliary system for the new variables $(q, \sigma)$

$$
\left\{\begin{array}{l}
\partial_{t} q=i\left(2 t^{2}\right)^{-1} \Delta q+t^{-2}(Q(s, q)+Q(\sigma, W))+i t^{-1} B_{0 S} q \\
+i t^{-1} B_{S}(w, w) q+i t^{-1}\left(2 B_{S}(W, q)+B_{S}(q, q)\right) W-R_{1}(W, S) \\
\partial_{t} \sigma=t^{-2}(s \cdot \nabla \sigma+\sigma \cdot \nabla S)-t^{-1} \nabla\left(2 B_{L}(W, q)+B_{L}(q, q)\right)-R_{2}(W, S),
\end{array}\right.
$$


where the remainders $R_{1}(W, S)$ and $R_{2}(W, S)$ are defined by

$$
\begin{gathered}
R_{1}(W, S)=\partial_{t} W-i\left(2 t^{2}\right)^{-1} \Delta W-t^{-2} Q(S, W)-i t^{-1}\left(B_{0 S}+B_{S}(W, W)\right) W \\
R_{2}(W, S)=\partial_{t} S-t^{-2} S \cdot \nabla S+t^{-1} \nabla B_{0 L}+t^{-1} \nabla B_{L}(W, W)
\end{gathered}
$$

and the dependence of the remainders on $B_{0}$ has been omitted in the notation. For technical reasons, it is useful to consider also a partly linearized version of the system (1.23), namely

$$
\left\{\begin{array}{l}
\partial_{t} q^{\prime}=i\left(2 t^{2}\right)^{-1} \Delta q^{\prime}+t^{-2}\left(Q\left(s, q^{\prime}\right)+Q(\sigma, W)\right)+i t^{-1} B_{0 S} q^{\prime} \\
+i t^{-1} B_{S}(w, w) q^{\prime}+i t^{-1}\left(2 B_{S}(W, q)+B_{S}(q, q)\right) W-R_{1}(W, S) \\
\partial_{t} \sigma^{\prime}=t^{-2}\left(s \cdot \nabla \sigma^{\prime}+\sigma \cdot \nabla S\right)-t^{-1} \nabla\left(2 B_{L}(W, q)+B_{L}(q, q)\right)-R_{2}(W, S) .
\end{array}\right.
$$

The construction of solutions $(w, s)$ of the auxiliary system (1.20) defined for large time and with prescribed asymptotic behaviour $(W, S)$ proceeds in two steps. The first step consists in solving the system $(1.23)$ for $(q, \sigma)$ tending to zero at infinity under suitable boundedness properties of $B_{0}$ and $(W, S)$ and suitable time decay properties of the remainders $R_{1}(W, S)$ and $R_{2}(W, S)$, by a minor variation of the method used in I. That method consists in first solving the linearized system (1.26) for $\left(q^{\prime}, \sigma^{\prime}\right)$ with given $(q, \sigma)$, and then showing that the map $(q, \sigma) \rightarrow\left(q^{\prime}, \sigma^{\prime}\right)$ thereby defined has a fixed point, by the use of a contraction method. The second step consists in constructing $(W, S)$ with $W(t)$ tending to $w_{+}$as $t \rightarrow \infty$ and satisfying the required boundedness and decay properties. This is done by solving the auxiliary system (1.20) by iteration to second order as in I and then adding to $W$ an additional term of the same form as that used in [8]. The detailed form of $(W, S)$ thereby obtained is too complicated to be given here and will be given in Section 3 below (see (3.25)-(3.29) and (3.31)).

Once the system $(1.20)$ is solved for $(w, s)$, one can proceed therefrom to the construction of a solution $(u, A)$ of the original WS system. One first defines the phases $\varphi$ and $\phi$ such that $s=\nabla \varphi$ and $S=\nabla \phi$ and one reconstructs $(u, A)$ from $(w, \varphi)$ by (1.11) (1.3) (1.5), thereby obtaining a solution of the WS system defined for large time and with prescribed asymptotic behaviour. The modified wave operator for the WS system is then defined as the map $\Omega:\left(u_{+}, A_{+}, \dot{A}_{+}\right) \rightarrow(u, A)$.

The main result of this paper is the construction of $(u, A)$ from $\left(u_{+}, A_{+}, \dot{A}_{+}\right)$as described above, together with the asymptotic properties of $(u, A)$ that follow from 
that construction. It will be stated below in full mathematical detail in Proposition 4.1. We give here a heuristic preview of that result, stripped from most technicalities. We set $\beta=\beta_{0}=1 / 3$ for definiteness.

Proposition 1.1. Let $\beta_{0}=\beta=1 / 3$. Let $\left(u_{+}, A_{+}, \dot{A}_{+}\right)$be such that $w_{+}=$ $F u_{+} \in H^{k_{+}}$for sufficiently large $k_{+}$, that $\left(A_{+}, \dot{A}_{+}\right)$be sufficiently regular, and that $\left(F A_{+}, F \dot{A}_{+}\right)$be sufficiently small near $\xi=0$. Let $(W, S)$ be the approximate solution of the system (1.20) defined by (3.25)-(3.29) (3.31). Then

(1) There exists $T=T\left(u_{+}, A_{+}, \dot{A}_{+}\right), 1 \leq T<\infty$, such that the auxiliary system (1.20) has a unique solution $(w, s)$ in a suitable space, defined for $t \geq T$ and such that $(w-W, s-S)$ tends to zero in suitable norms when $t \rightarrow \infty$.

(2) There exists $\varphi$ and $\phi$ such that $s=\nabla \varphi, S=\nabla \phi, \phi(1)=0$ and such that $\varphi-\phi$ tends to zero in suitable norms when $t \rightarrow \infty$. Define $(u, A)$ by (1.11) (1.3) (1.5). Then $(u, A)$ solves the system (1.1) (1.2) for $t \geq T$ and $(u, A)$ behaves asymptotically as $\left(M D \exp (-i \phi) W, A_{0}+A_{1}\left(|D W|^{2}\right)\right)$ in the sense that the difference tends to zero in suitable norms (for which each term separately is $O(1)$ ) when $t \rightarrow \infty$.

The unspecified condition that $\left(F A_{+}, F \dot{A}_{+}\right)$be sufficiently small near $\xi=0$ can be shown to follow from more intuitive conditions in $x$-space, consisting of decay conditions at infinity in space, and, depending on the values of the parameters defining the relevant function spaces, of some moment conditions on $\left(A_{+}, \dot{A}_{+}\right)$.

This paper relies on a large amount of material from I. In order to bring out the structure while keeping duplication to a minimum, we give without proof a shortened logically self-sufficient sequence of those intermediate results from I that are needed, and we provide a full exposition only for the parts that are new as compared with I. When quoting I, we shall use the notation (I.p.q) for equation (p.q) of I and Item I.p.q for Item p.q of I, such as Lemma, Proposition, etc.

The remaining part of this paper is organized as follows. In Section 2 we collect notation and some estimates of a general nature. In Section 3, we study the Cauchy problem at infinity for the auxiliary system (1.20). We recall from I the existence results of solutions under suitable boundedness properties of $(W, S)$ and suitable decay properties of the remainders, with the appropriate modifications (Proposition 3.1). We then define $(W, S)$ and prove that they satisfy the previous properties, concentrating on the terms in the remainders that are new as compared with I (Proposition 3.2). We then discuss the assumptions on $\left(F A_{+}, F \dot{A}_{+}\right)$at $\xi=0$ mentioned above. 
Finally in Section 4, we construct the wave operators for the WS system (1.1) (1.2) and we derive the asymptotic properties of the solution $(u, A)$ in their range that follow from the previous estimates (Proposition 4.1).

\section{Notation and preliminary estimates}

In this section we introduce some notation and we collect a number of estimates which will be used throughout this paper. We denote by $\|\cdot\|_{r}$ the norm in $L^{r} \equiv$ $L^{r}\left(I^{3}\right)$ and we define $\delta(r)=3 / 2-3 / r$. For any interval $I$ and any Banach space $X$ we denote by $\mathcal{C}(I, X)$ the space of strongly continuous functions from $I$ to $X$ and by $L^{\infty}(I, X)$ the space of measurable essentially bounded functions from $I$ to $X$. For real numbers $a$ and $b$ we use the notation $a \vee b=\operatorname{Max}(a, b)$ and $a \wedge b=\operatorname{Min}(a, b)$. In the estimates of solutions of the relevant equations we shall use the letter $\mathrm{C}$ to denote constants, possibly different from an estimate to the next, depending on various parameters but not on the solutions themselves or on their initial data. We shall use the notation $C\left(a_{1}, a_{2}, \cdots\right)$ for estimating functions, also possibly different from an estimate to the next, depending on suitable norms $a_{1}, a_{2}, \cdots$ of the solutions or of their initial data.

We shall use the Sobolev spaces $\dot{H}_{r}^{k}$ and $H_{r}^{k}$ defined for $-\infty<k<+\infty, 1 \leq$ $r \leq \infty$ by

$$
\dot{H}_{r}^{k}=\left\{u:\left\|u ; \dot{H}_{r}^{k}\right\| \equiv\left\|\omega^{k} u\right\|_{r}<\infty\right\}
$$

and

$$
H_{r}^{k}=\left\{u:\left\|u ; H_{r}^{k}\right\| \equiv\left\|<\omega>^{k} u\right\|_{r}<\infty\right\}
$$

where $\omega=(-\Delta)^{1 / 2}$ and $\langle\cdot\rangle=\left(1+|\cdot|^{2}\right)^{1 / 2}$. The subscript $r$ will be omitted if $r=2$.

We shall look for solutions of the auxiliary system (1.20) in spaces of the type $\mathcal{C}\left(I, X^{k, \ell}\right)$ where $I$ is an interval and

$$
X^{k, \ell}=H^{k} \oplus \omega^{-1} H^{\ell}
$$

namely

$$
X^{k, \ell}=\left\{(w, s): w \in H^{k}, \nabla s \in H^{\ell}\right\}
$$

where it is understood that $\nabla s \in L^{2}$ includes the fact that $s \in L^{6}$, and we shall use the notation

$$
\left\|w ; H^{k}\right\|=|w|_{k}
$$


We shall use extensively the following Sobolev inequalities, stated here in $I R^{n}$, but to be used only for $n=3$.

Lemma 2.1. Let $1<q, r<\infty, 1<p \leq \infty$ and $0 \leq j<k$. If $p=\infty$, assume that $k-j>n / r$. Let $\sigma$ satisfy $j / k \leq \sigma \leq 1$ and

$$
n / p-j=(1-\sigma) n / q+\sigma(n / r-k) .
$$

Then the following inequality holds

$$
\left\|\omega^{j} u\right\|_{p} \leq C\|u\|_{q}^{1-\sigma}\left\|\omega^{k} u\right\|_{r}^{\sigma} .
$$

The proof follows from the Hardy-Littlewood-Sobolev (HLS) inequality ([2], p. 117) (from the Young inequality if $p=\infty$ ), from Paley-Littlewood theory and interpolation.

We shall also use extensively the following Leibnitz and commutator estimates.

Lemma 2.2. Let $1<r, r_{1}, r_{3}<\infty$ and

$$
1 / r=1 / r_{1}+1 / r_{2}=1 / r_{3}+1 / r_{4} .
$$

Then the following estimates hold

$$
\left\|\omega^{m}(u v)\right\|_{r} \leq C\left(\left\|\omega^{m} u\right\|_{r_{1}}\|v\|_{r_{2}}+\left\|\omega^{m} v\right\|_{r_{3}}\|u\|_{r_{4}}\right)
$$

for $m \geq 0$, and

$$
\left\|\left[\omega^{m}, u\right] v\right\|_{r} \leq C\left(\left\|\omega^{m} u\right\|_{r_{1}}\|v\|_{r_{2}}+\left\|\omega^{m-1} v\right\|_{r_{3}}\|\nabla u\|_{r_{4}}\right)
$$

for $m \geq 1$, where $[$,$] denotes the commutator.$

The proof of those estimates is given in [3] [4] with $\omega$ replaced by $\langle\omega\rangle$ and follows therefrom by a scaling argument.

We next give some estimates of $B_{0 L}, B_{0 S}, B_{S}$ and $B_{L}$ defined by (1.16) (1.17). It follows immediately from (1.16) (1.17) that

$$
\left\|\omega^{m} B_{0 L}\right\|_{2} \leq\left(2 t_{0}^{\beta_{0}}\right)^{m-p}\left\|\omega^{p} B_{0 L}\right\|_{2} \leq\left(2 t^{\beta_{0}}\right)^{m-p}\left\|\omega^{p} B_{0}\right\|_{2}
$$


for $m \geq p$ and

$$
\left\|\omega^{m} B_{0 S}\right\|_{2} \leq t^{\beta_{0}(m-p)}\left\|\omega^{p} B_{0 S}\right\|_{2} \leq t^{\beta_{0}(m-p)}\left\|\omega^{p} B_{0}\right\|_{2}
$$

for $m \leq p$. Similar estimates hold for $B_{L}, B_{S}$ with $\beta_{0}$ replaced by $\beta$. On the other hand it follows from (1.15) that

$$
\left\|\omega^{m+1} B_{1}\left(w_{1}, w_{2}\right)\right\|_{2} \leq I_{m}\left(\left\|\omega^{m}\left(\bar{w}_{1} w_{2}\right)\right\|_{2}\right)
$$

where $I_{m}$ is defined by

$$
\left(I_{m}(f)\right)(t)=\int_{1}^{\infty} d \nu \nu^{-m-3 / 2} f(\nu t) .
$$

We finally collect some estimates of the solutions of the free wave equation $\square A_{0}=0$ with initial data $\left(A_{+}, \dot{A}_{+}\right)$at time zero, given by (1.4).

Lemma 2.3. Let $k \geq 0$. Let $A_{+}$and $\dot{A}_{+}$satisfy the conditions

$$
A_{+}, \omega^{-1} \dot{A}_{+} \in H^{k} \quad, \quad \nabla^{2} A_{+}, \nabla \dot{A}_{+} \in H_{1}^{k}
$$

Then the following estimate holds :

$$
\left\|\omega^{m} A_{0}\right\|_{r} \leq b_{0} t^{-1+2 / r} \quad \text { for } 2 \leq r \leq \infty,
$$

for $0 \leq m \leq k$ and for all $t>0$, where $b_{0}$ depends on $\left(A_{+}, \dot{A}_{+}\right)$through the norms associated with (2.10).

The estimate (2.11) can be expressed in an equivalent form in terms of $B_{0}$ defined by (1.13), namely

$$
\left\|\omega^{m} B_{0}\right\|_{r} \leq b_{0} t^{m-1 / r} \quad \text { for } 2 \leq r \leq \infty
$$

Furthermore, it follows from (1.17) and (2.12) that

$$
\left\|\omega^{m} B_{0 L}\right\|_{r} \leq\left\|F^{-1} \chi\right\|_{1}\left\|\omega^{m} B_{0}\right\|_{r} \leq C b_{0} t^{m-1 / r}
$$

where we have used the Young inequality and the fact that the $L^{1}$-norm of $F^{-1} \chi$ is invariant under the rescaling of $\xi$ by $t^{\beta_{0}}$ which occurs in (1.17). From (2.12) (2.13) and (1.16) it follows that also

$$
\left\|\omega^{m} B_{0 S}\right\|_{r} \leq C b_{0} t^{m-1 / r}
$$

In the applications, the estimate (2.12) will be used mostly through its consequence $(2.14)$. 


\section{Cauchy problem at infinity for the auxiliary system}

In this section, we solve the Cauchy problem at infinity for the auxiliary system (1.20) in the difference form (1.23). We first solve the system $(1.23)$ for $(q, \sigma)$ tending to zero at infinity under suitable boundedness properties of $\left(B_{0}, W, S\right)$ and suitable time decay properties of the remainders $R_{1}(W, S)$ and $R_{2}(W, S)$. We then construct $(W, S)$ with $W(t)$ tending to $w_{+}=F u_{+}$as $t \rightarrow \infty$ and satisfying the required boundedness and decay properties. The method closely follows that of Sections 6 and 7 of I.

We first estimate a single solution of the linearized auxiliary system (1.26) at the level of regularity where we shall eventually solve the auxiliary system (1.20). The following lemma is basically Lemma I.6.1, restricted to the case where $1<k<3 / 2$, and sharpened in order to take into account the fact that the $W$ used in this paper is less regular than that used in I (compare (3.1) below with (I.6.1)).

Lemma 3.1. Let $1<k<\ell<3 / 2$ and $\beta>0$. Let $T \geq 1$ and $I=[T, \infty)$. Let $B_{0}$ satisfy the estimate (2.12) for $0 \leq m \leq k$. Let $\left.(U(1 / t)) W, S\right) \in \mathcal{C}\left(I, X^{k+1, \ell+1}\right) \cap$ $\mathcal{C}^{1}\left(I, X^{k, \ell}\right)$ and let $W$ satisfy

$$
\operatorname{Sup}_{t \in I}\left\{\|W\|_{\infty} \vee\left\|W ; H^{3 / 2}\right\| \vee t^{1 / 2-k}\left\|W ; \dot{H}^{k+1}\right\|\right\} \leq a<\infty
$$

Let $(q, \sigma),\left(q^{\prime}, \sigma^{\prime}\right) \in \mathcal{C}\left(I, X^{k, \ell}\right)$ with $q \in L^{\infty}\left(I, H^{k}\right) \cap L^{2}\left(I, L^{2}\right)$ and let $\left(q^{\prime}, \sigma^{\prime}\right)$ be a solution of the system (1.26) in I. Then the following estimates hold for all $t \in I$ :

$$
\begin{gathered}
\left|\partial_{t}\left\|q^{\prime}\right\|_{2}\right| \leq C\left\{t^{-2} a\|\nabla \sigma\|_{2}+t^{-1-\beta} a^{2} I_{0}\left(\|q\|_{2}\right)\right. \\
\left.+t^{-1} a I_{-1}\left(\|q\|_{2}\|q\|_{3}\right)\right\}+\left\|R_{1}(W, S)\right\|_{2}, \\
\left|\partial_{t}\left\|\omega^{k} q^{\prime}\right\|_{2}\right| \leq C\left\{b_{0}\left(\left\|\omega^{k-1} q^{\prime}\right\|_{2}+t^{k-1-\delta / 3}\left\|q^{\prime}\right\|_{r}\right)\right. \\
+t^{-2} a\left(\left\|\omega^{k} \nabla \sigma\right\|_{2}+t^{k-1 / 2}\|\sigma\|_{\infty}\right) \\
+t^{-2}\left(\|\nabla s\|_{\infty}+\left\|\omega^{3 / 2} \nabla s\right\|_{2}\right)\left\|\omega^{k} q^{\prime}\right\|_{2} \\
+t^{-1} a^{2}\left(I_{k-1}\left(\left\|\omega^{k-1} q\right\|_{2}\right)+\left\|\omega^{1 / 2} q^{\prime}\right\|_{2}\right) \\
+t^{-1} a\left(I_{k-1}\left(\left\|\omega^{k} q\right\|_{2}\|q\|_{3}\right)+I_{1 / 2}\left(\left\|\omega^{1 / 2} q\right\|_{2}\right)\left\|\omega^{k} q^{\prime}\right\|_{2}\right) \\
\left.+t^{-1} I_{1 / 2}\left(\|\nabla q\|_{2}^{2}\right)\left\|\omega^{k} q^{\prime}\right\|_{2}\right\}+\left\|\omega^{k} R_{1}(W, S)\right\|_{2}
\end{gathered}
$$


where $s=S+\sigma$ and $0<\delta=\delta(r) \leq k$.

$$
\begin{aligned}
& \left|\partial_{t}\left\|\omega^{m} \nabla \sigma^{\prime}\right\|_{2}\right| \leq C t^{-2}\left\{\|\nabla s\|_{\infty}\left\|\omega^{m} \nabla \sigma^{\prime}\right\|_{2}+\left\|\omega^{m} \nabla s\right\|_{2}\left\|\nabla \sigma^{\prime}\right\|_{\infty}\right. \\
& \left.+\left\|\omega^{m} \nabla \sigma\right\|_{2}\|\nabla S\|_{\infty}+\|\sigma\|_{\infty}\left\|\omega^{m} \nabla^{2} S\right\|_{2}\right\} \\
& +C\left\{t^{-1+\beta(m+1)} a I_{0}\left(\|q\|_{2}\right)+t^{-1+\beta(m+5 / 2)} I_{-3 / 2}\left(\|q\|_{2}^{2}\right)\right\} \\
& +\left\|\omega^{m} \nabla R_{2}(W, S)\right\|_{2}
\end{aligned}
$$

for $0 \leq m \leq \ell$

$$
\begin{aligned}
& \left|\partial_{t}\left\|\nabla \sigma^{\prime}\right\|_{2}\right| \leq C t^{-2}\left\{\|\nabla s\|_{\infty}\left\|\nabla \sigma^{\prime}\right\|_{2}+\|\nabla \sigma\|_{2}\left(\|\nabla S\|_{\infty}+\left\|\omega^{3 / 2} \nabla S\right\|_{2}\right)\right\} \\
& \left.\quad+C\left\{t^{-1+\beta} a I_{0}\left(\|q\|_{2}\right)+t^{-1+5 \beta / 2} I_{-3 / 2}\left(\|q\|_{2}^{2}\right)\right\}+\left\|\nabla R_{2}(W, S)\right\|_{2} \quad \text { (3.4) }\right)_{0}
\end{aligned}
$$

Proof. (3.2) is identical with (I.6.2) and is proved in the same way. In order to prove (3.3), we start from (cf. I.6.9))

$$
\begin{aligned}
& \left|\partial_{t}\left\|\omega^{k} q^{\prime}\right\|_{2}\right| \leq t^{-1}\left\|\left[\omega^{k}, B_{0 S}\right] q^{\prime}\right\|_{2}+t^{-2}\left(\left\|\left[\omega^{k}, s\right] \cdot \nabla q^{\prime}\right\|_{2}\right. \\
& \left.+\left\|(\nabla \cdot s) \omega^{k} q^{\prime}\right\|_{2}+\left\|\omega^{k}\left((\nabla \cdot s) q^{\prime}\right)\right\|_{2}+\left\|\omega^{k} Q(\sigma, W)\right\|_{2}\right) \\
& t^{-1}\left(\left\|\left[\omega^{k}, B_{S}(w, w)\right] q^{\prime}\right\|_{2}+\left\|\omega^{k}\left(2 B_{S}(W, q)+B_{S}(q, q)\right) W\right\|_{2}\right) \\
& +\left\|\omega^{k} R_{1}(W, S)\right\|_{2}
\end{aligned}
$$

and we estimate the various terms in the RHS successively.

The contribution of $B_{0}$ is estimated exactly as in I and yields

$$
\left\|\left[\omega^{k}, B_{0 S}\right] q^{\prime}\right\|_{2} \leq C b_{0}\left(t\left\|\omega^{k-1} q^{\prime}\right\|_{2}+t^{k-\delta / 3}\left\|q^{\prime}\right\|_{r}\right)
$$

The contribution of $Q\left(s, q^{\prime}\right)$ is estimated by Lemmas 2.1 and 2.2 as

$$
\begin{aligned}
& \left\|\left[\omega^{k}, s\right] \cdot \nabla q^{\prime}\right\|_{2}+\left\|(\nabla \cdot s) \omega^{k} q^{\prime}\right\|_{2}+\left\|\omega^{k}\left((\nabla \cdot s) q^{\prime}\right)\right\|_{2} \\
& \leq C\left(\|\nabla s\|_{\infty}+\left\|\omega^{3 / 2} \nabla s\right\|_{2}\right)\left\|\omega^{k} q^{\prime}\right\|_{2}
\end{aligned}
$$

in the same way as in I, in the case $k<3 / 2$.

The contribution of $Q(\sigma, W)$ is estimated by Lemma 2.1 and 2.2 as

$$
\begin{gathered}
\left\|\omega^{k} Q(\sigma, W)\right\|_{2} \leq C\left(\|\sigma\|_{\infty}\left\|\omega^{k} \nabla W\right\|_{2}+\left\|\omega^{k} \sigma\right\|_{6}\|\nabla W\|_{3}\right. \\
\left.+\left\|\omega^{k} \nabla \sigma\right\|_{2}\|W\|_{\infty}+\|\nabla \sigma\|_{r}\left\|\omega^{k} W\right\|_{3 / k}\right)
\end{gathered}
$$


with $\delta(r)=k$,

$$
\begin{aligned}
& \cdots \leq C\left(\|\sigma\|_{\infty}\left\|\omega^{k} \nabla W\right\|_{2}+\left\|\omega^{k} \nabla \sigma\right\|_{2}\left(\|W\|_{\infty}+\left\|\omega^{k} W\right\|_{3 / k}\right)\right) \\
& \leq C a\left(t^{k-1 / 2}\|\sigma\|_{\infty}+\left\|\omega^{k} \nabla \sigma\right\|_{2}\right)
\end{aligned}
$$

by (3.1).

The contribution of $B_{S}$ with $w=W+q$ yields a number of terms which we order by increasing powers of $q, q^{\prime}$. We first expand

$$
B_{S}(w, w)=B_{S}(W, W)+2 B_{S}(W, q)+B_{S}(q, q) .
$$

By Lemmas 2.1 and 2.2, we estimate

$$
\begin{gathered}
\left\|\left[\omega^{k}, B_{S}(W, W)\right] q^{\prime}\right\|_{2} \leq C(\| \\
\left.\nabla B_{S}(W, W)\left\|_{3 / \varepsilon}+\right\| \omega^{k} B_{S}(W, W) \|_{3 /(k-1+\varepsilon)}\right) \\
\times\left\|\omega^{k-1+\varepsilon} q^{\prime}\right\|_{2}
\end{gathered}
$$

for $\varepsilon>0$. Taking $\varepsilon=3 / 2-k$ yields

$$
\begin{aligned}
& \cdots \leq C\left\|\omega^{k+1} B_{1}(W, W)\right\|_{2}\left\|\omega^{1 / 2} q^{\prime}\right\|_{2} \\
& \leq C I_{k}\left(\left\|\omega^{k} W\right\|_{2}\|W\|_{\infty}\right)\left\|\omega^{1 / 2} q^{\prime}\right\|_{2} \\
& \leq C a^{2}\left\|\omega^{1 / 2} q^{\prime}\right\|_{2}
\end{aligned}
$$

by Lemma 2.2 again and by (2.8) (3.1).

In a similar way, we estimate by Lemmas $2.1,2.2$ and by (2.8)

$\left\|\left[\omega^{k}, B_{S}(W, q)\right] q^{\prime}\right\|_{2} \leq C\left(\left\|\nabla B_{S}(W, q)\right\|_{3}\left\|\omega^{k-1} q^{\prime}\right\|_{6}+\left\|\omega^{k} B_{S}(W, q)\right\|_{3 / k}\left\|q^{\prime}\right\|_{r}\right)$ with $\delta(r)=k$,

$$
\begin{aligned}
& \cdots \leq C\left\|\omega^{3 / 2} B_{1}(W, q)\right\|_{2}\left\|\omega^{k} q^{\prime}\right\|_{2} \\
& \leq C I_{1 / 2}\left(\|W\|_{\infty}\left\|\omega^{1 / 2} q\right\|_{2}+\left\|\omega^{1 / 2} W\right\|_{6}\|q\|_{3}\right)\left\|\omega^{k} q^{\prime}\right\|_{2} \\
& \leq C a I_{1 / 2}\left(\left\|\omega^{1 / 2} q\right\|_{2}\right)\left\|\omega^{k} q^{\prime}\right\|_{2} .
\end{aligned}
$$

In a similar way, we estimate

$$
\begin{aligned}
& \left\|\left[\omega^{k}, B_{S}(q, q)\right] q^{\prime}\right\|_{2} \leq C\left(\left\|\nabla B_{S}(q, q)\right\|_{3}+\left\|\omega^{k} B_{S}(q, q)\right\|_{3 / k}\right)\left\|\omega^{k} q^{\prime}\right\|_{2} \\
& \leq C\left\|\omega^{3 / 2} B_{1}(q, q)\right\|_{2}\left\|\omega^{k} q^{\prime}\right\|_{2} \\
& \leq C I_{1 / 2}\left(\left\|\omega^{1 / 2} q\right\|_{3}\|q\|_{6}\right)\left\|\omega^{k} q^{\prime}\right\|_{2} \\
& \leq C I_{1 / 2}\left(\|\nabla q\|_{2}^{2}\right)\left\|\omega^{k} q^{\prime}\right\|_{2} .
\end{aligned}
$$


We next estimate in a similar way

$$
\begin{aligned}
& \left\|\omega^{k}\left(B_{S}(W, q) W\right)\right\|_{2} \leq C\left\|\omega^{k} B_{1}(W, q)\right\|_{2}\left(\|W\|_{\infty}+\left\|\omega^{k} W\right\|_{3 / k}\right) \\
& \leq C a\left\|\omega^{k} B_{1}(W, q)\right\|_{2} \\
& \leq C a I_{k-1}\left(\left(\|W\|_{\infty}+\left\|\omega^{k-1} W\right\|_{3 /(k-1)}\right)\left\|\omega^{k-1} q\right\|_{2}\right) \\
& \leq C a^{2} I_{k-1}\left(\left\|\omega^{k-1} q\right\|_{2}\right) .
\end{aligned}
$$

Finally, we estimate in a similar way

$$
\begin{aligned}
& \left\|\omega^{k}\left(B_{S}(q, q) W\right)\right\|_{2} \leq C\left\|\omega^{k} B_{1}(q, q)\right\|_{2}\left(\|W\|_{\infty}+\left\|\omega^{k} W\right\|_{3 / k}\right) \\
& \leq C a I_{k-1}\left(\left\|\omega^{k-1}|q|^{2}\right\|_{2}\right) \\
& \leq C a I_{k-1}\left(\left\|\omega^{k} q\right\|_{2}\|q\|_{3}\right) .
\end{aligned}
$$

Substituting (3.6)-(3.8) and (3.10)-(3.14) into (3.5) yields (3.3).

The estimates (3.4) and (3.4) of $\sigma^{\prime}$ are identical with (I.6.4) and (I.6.4) $)_{0}$ and have exactly the same proof. The additional term with $B_{0 L}$ in the equation for $\sigma^{\prime}$ is included in $R_{2}(W, S)$ and does not appear explicitly at this stage.

From there on, the treatment of the Cauchy problem at infinity for the auxiliary system follows that given in I verbatim. We need to estimate the difference of two solutions of the linearized auxiliary system (1.26), and that estimate, given by Lemma I.6.2, requires no modification because it uses regularity properties of $W$ which are weaker than (3.1). We then solve the Cauchy problem first for the linearized auxiliary system (1.26) with finite initial time by Proposition I.6.1, then at infinity by Proposition I.6.2, and then for the auxiliary system (1.20) or (1.23) by a contraction method, by Proposition I.6.3, part (2). The only difference in the proof of Propositions I.6.2 and I.6.3 is due to the term

$$
t^{-2} a t^{k-1 / 2}\|\sigma\|_{\infty}
$$

in (3.3), which did not appear in Lemma I.6.1, and which is due to the fact that the assumption (3.1) is weaker than (I.6.1). That term generates an additional term

$$
\text { a } Z t^{-1-\lambda-3(1-\beta) / 2}
$$

in the RHS of (I.6.59), with time decay strictly better than $t^{-1-\lambda}$ and therefore harmless. 
We now state the first main result of this section, which corresponds to Proposition I.6.3, part (2).

Proposition 3.1. Let $1<k<3 / 2<\ell$. Let $\lambda_{0}, \lambda$ and $\beta$ satisfy the conditions

$$
\begin{gathered}
\lambda>0, \quad(1<) \lambda+k<\lambda_{0}, \\
0<\beta<2 / 3 \quad, \quad \beta(\ell+1)<\lambda_{0} .
\end{gathered}
$$

Let $\left(A_{+}, \dot{A}_{+}\right)$satisfy the conditions (2.10). Let $(U(1 / t) W, S) \in \mathcal{C}\left([1, \infty), X^{k+1, \ell+1}\right) \cap$ $\mathcal{C}^{1}\left([1, \infty), X^{k, \ell}\right)$ and let $(W, S)$ satisfy the estimates

$$
\begin{gathered}
\operatorname{Sup}_{t \geq 1}\left\{\|W\|_{\infty} \vee\left\|W ; H^{3 / 2}\right\| \vee t^{1 / 2-k}\left\|W ; \dot{H}^{k+1}\right\|\right\} \leq a<\infty, \\
\left\|\omega^{m} \nabla S\right\|_{2} \leq b t^{1-\eta+\beta(m-3 / 2)}
\end{gathered}
$$

for some $\eta>0$ and for $0 \leq m \leq \ell+1$,

$$
\begin{gathered}
\left\|R_{1}(W, S)\right\|_{2} \leq c_{0} t^{-1-\lambda_{0}}, \\
\left\|\omega^{k} R_{1}(W, S)\right\|_{2} \leq c_{1} t^{-1-\lambda}, \\
\left\|\omega^{m} \nabla R_{2}(W, S)\right\|_{2} \leq c_{2} t^{-1-\lambda_{0}+\beta(m+1)} \text { for } 0 \leq m \leq \ell .
\end{gathered}
$$

Then there exists $T, 1 \leq T<\infty$ and positive constants $Y_{0}, Y$ and $Z$, depending on $\left(A_{+}, \dot{A}_{+}\right)$through the norms in (2.10) and depending on $k, \ell, \beta, \lambda_{0}, \lambda, a, b, c_{0}, c_{1}$ and $c_{2}$ such that the auxiliary system (1.20) has a unique solution $(w, s) \in \mathcal{C}\left(I, X^{k, \ell}\right)$, where $I=[T, \infty)$, satisfying the estimates

$$
\begin{gathered}
\|w-W\|_{2} \leq Y_{0} t^{-\lambda_{0}}, \\
\left\|\omega^{k}(w-W)\right\|_{2} \leq Y t^{-\lambda}, \\
\left\|\omega^{m} \nabla(s-S)\right\|_{2} \leq Z t^{-\lambda_{0}+\beta(m+1)} \text { for } 0 \leq m \leq \ell,
\end{gathered}
$$

for all $t \in I$.

We now turn to the construction of approximate solutions $(W, S)$ of the system (1.20) satisfying the assumptions of Proposition 3.1 and in particular the estimates (3.17) (3.18) of (W,S) and the estimates (3.19)-(3.21) of the remainders. In I we took for $(W, S)$ the second order approximate solution of the system (1.20) in an iterative scheme not taking into account the terms containing $B_{0}$, thereby ending with an 
explicit $B_{0} W$ term in the remainder $R_{1}(W, S)$. Here, following [8], we improve that asymptotic form by adding one more term in $W$, so as to partly cancel the $B_{0 S} W$ term in $R_{1}(W, S)$. Thus we define

$$
W=w_{0}+w_{1}+w_{2} \equiv W_{1}+w_{2} \quad, \quad S=s_{0}+s_{1}
$$

where $w_{0}, s_{0}, w_{1}, s_{1}$ are the same as in I, namely

$$
\begin{gathered}
w_{0}=U^{*}(1 / t) w_{+}, \\
s_{0}(t)=-\int_{1}^{t} d t^{\prime} t^{\prime-1} \nabla B_{L}\left(w_{0}\left(t^{\prime}\right), w_{0}\left(t^{\prime}\right)\right), \\
w_{1}(t)=-U^{*}(1 / t) \int_{t}^{\infty} d t^{\prime} t^{\prime-2} U\left(1 / t^{\prime}\right) Q\left(s_{0}\left(t^{\prime}\right), w_{0}\left(t^{\prime}\right)\right), \\
s_{1}(t)=-\int_{t}^{\infty} d t^{\prime} t^{\prime-2} s_{0}\left(t^{\prime}\right) \cdot \nabla s_{0}\left(t^{\prime}\right)+2 \int_{t}^{\infty} d t^{\prime} t^{\prime-1} \nabla B_{L}\left(w_{0}\left(t^{\prime}\right), w_{1}\left(t^{\prime}\right)\right) .
\end{gathered}
$$

In order to partly cancel $B_{0 S} W$ in $R_{1}(W, S)$, we take $w_{2}=h w_{0}$, thereby obtaining a linear contribution of $w_{2}$ to $R_{1}(W, S)$

$$
\begin{aligned}
\left(\partial_{t}-i\left(2 t^{2}\right)^{-1} \Delta\right) w_{2}= & h\left(\partial_{t}-i\left(2 t^{2}\right)^{-1} \Delta\right) w_{0}-i t^{-2} \nabla h \cdot \nabla w_{0}+\left(\partial_{t} h\right) w_{0} \\
& -i\left(2 t^{2}\right)^{-1}(\Delta h) w_{0}
\end{aligned}
$$

The first term in the RHS is small, actually zero, by the choice of $w_{0}$. We use the last term in the RHS to cancel the main contribution $B_{0 S} w_{0}$ of $B_{0 S} W$ by making the choice

$$
w_{2}=h w_{0} \quad, \quad h=-2 t \Delta^{-1} B_{0 S} .
$$

Note that because of the short range cut-off in $B_{0 S}, h$ is well defined, actually $h \in \mathcal{C}\left([1, \infty), H^{k+2}\right)$. With that choice, the remainders become

$$
R_{i}(W, S)=R_{i 0}(W, S)+R_{i \nu}(W, S) \quad i=1,2,
$$

where $R_{i 0}(W, s)$ are the parts not containing $w_{2}$ or $B_{0 L}$, namely

$$
\begin{gathered}
R_{10}(W, S)=-t^{-2}\left(Q\left(S, w_{1}\right)+Q\left(s_{1}, w_{0}\right)\right) \\
-i t^{-1}\left(B_{0 S} w_{1}+B_{S}\left(W_{1}, W_{1}\right) W_{1}\right) \\
R_{20}(W, S)=-t^{-2}\left(s_{0} \cdot \nabla s_{1}+s_{1} \cdot \nabla s_{0}+s_{1} \cdot \nabla s_{1}\right)+t^{-1} \nabla B_{L}\left(w_{1}, w_{1}\right),
\end{gathered}
$$

while $R_{i \nu}(W, S)$ are the parts containing $w_{2}$ or $B_{0 L}$, namely

$$
\begin{aligned}
R_{1 \nu}(W, S)= & -t^{-2} Q\left(S, w_{2}\right)-i t^{-1} B_{0 S} w_{2}+\left(\partial_{t} h\right) w_{0}-i t^{-2} \nabla h \cdot \nabla w_{0} \\
& -i t^{-1}\left(B_{S}(W, W) w_{2}+B_{S}\left(W+W_{1}, w_{2}\right) W_{1}\right)
\end{aligned}
$$




$$
R_{2 \nu}(W, S)=t^{-1} \nabla B_{0 L}+t^{-1} \nabla B_{L}\left(W+W_{1}, w_{2}\right) .
$$

The parts $R_{i 0}$ of the remainders are the remainders occurring in I, up to the replacement of $B_{0}$ by $B_{0 S}$ and the disappearance of the term $B_{0 S} w_{0}$, precisely the term which was responsible for the support condition in I. Up to a minor point (see below), $\left(W_{1}, S\right)$ and $R_{i 0}(W, S)$ have been estimated in I as follows (see Lemma I.7.1).

Lemma 3.2. Let $0<\beta<1, k_{+} \geq 3, w_{+} \in H^{k_{+}}$and $a_{+}=\left|w_{+}\right|_{k_{+}}$. Then the following estimates hold for all $t \geq 1$ :

$$
\begin{aligned}
& \left|w_{0}\right|_{k_{+}} \leq a_{+}, \\
& \left\|\omega^{m} s_{0}\right\|_{2} \leq \begin{cases}C a_{+}^{2} \ell n t & \text { for } 0 \leq m \leq k_{+} \\
C a_{+}^{2} t^{\beta\left(m-k_{+}\right)} & \text {for } m>k_{+},\end{cases} \\
& \left|w_{1}\right|_{k_{+}-1} \leq C a_{+}^{3} t^{-1}(1+\ln t), \\
& \left\|\omega^{m} s_{1}\right\|_{2} \leq\left\{\begin{array}{l}
C a_{+}^{4} t^{-1}(1+\ell n t)^{2} \quad \text { for } 0 \leq m \leq k_{+}-1 \\
C a_{+}^{4} t^{-1+\beta\left(m+1-k_{+}\right)}(1+\ell n t) \\
\quad \text { for } k_{+}-1<m<k_{+}-1+\beta^{-1}
\end{array}\right. \\
& \left\|\omega^{m} R_{20}(W, S)\right\|_{2} \leq\left\{\begin{array}{l}
C\left(a_{+}\right) t^{-3}(1+\ell n t)^{3} \quad \text { for } 0 \leq m \leq k_{+}-2 \\
C\left(a_{+}\right) t^{-3+\beta\left(m+2-k_{+}\right)}(1+\ell n t)^{2} \\
\quad \text { for } k_{+}-2<m<k_{+}-2+\beta^{-1},
\end{array}\right.
\end{aligned}
$$

Let in addition $0 \leq k \leq k_{+}-1$ and let $B_{0}$ satisfy the estimate (2.12) for $0 \leq m \leq k$. Then

$$
\begin{aligned}
\left\|\omega^{m} R_{10}(W, S)\right\|_{2} \leq & C\left(a_{+}\right)\left\{t^{-3}(1+\ell n t)^{2}+t^{-1-\beta\left(k_{+}-m+1\right)}\right\} \\
& +C b_{0} a_{+}^{3} t^{m-5 / 2}(1+\ell n t) \quad \text { for } 0 \leq m \leq k .
\end{aligned}
$$

Proof. The estimates (3.37)-(3.42) are those of Lemma I.7.1 except for the estimate of the term $t^{-1} B_{0 S} w_{1}$ in $R_{10}(W, S)$ which is responsible for the last term in $(3.42)$. 
We estimate that term by Lemma 2.1 and 2.2 and by (2.12) (3.39) as

$$
\begin{aligned}
\left\|\omega^{m} B_{0 S} w_{1}\right\|_{2} & \leq \begin{cases}C\left(\left\|\omega^{m} B_{0}\right\|_{2}\left\|w_{1}\right\|_{\infty}+\left\|B_{0 S}\right\|_{r}\left\|\omega^{m} w_{1}\right\|_{1 / m}\right) & \text { for } m \leq 1 / 2 \\
C\left(\left\|\omega^{m} B_{0}\right\|_{2}\left\|w_{1}\right\|_{\infty}+\left\|B_{0 S}\right\|_{\infty}\left\|\omega^{m} w_{1}\right\|_{2}\right) & \text { for } m>1 / 2\end{cases} \\
& \leq C b_{0} a_{+}^{3} t^{m-3 / 2}(1+\ell n t)
\end{aligned}
$$

for $0 \leq m \leq k$, and $1 / r=1 / 2-m$ for $m \leq 1 / 2$, which completes the proof of $(3.42)$.

We now turn to estimating $R_{i \nu}(W, S), i=1,2$. We first reduce that question to that of estimating $h$ and $B_{0 L}$, assuming for the moment a boundedness property of $w_{2}$ which is part of (3.17) and which we shall prove later. We define the auxiliary space

$$
Y=L^{\infty}\left([1, \infty), L^{\infty} \cap H^{3 / 2}\right)
$$

and we remark that for $k_{+}>5 / 2$, it follows from (3.37) (3.39) that $W_{1}=w_{0}+w_{1} \in$ $Y$. We can now state the estimates of $R_{i \nu}(W, S)$.

Lemma 3.3. Let $1<k<3 / 2$ and $k_{+} \geq 3$, let $w_{+} \in H^{k_{+}}$and let $a_{+}=\left|w_{+}\right|_{k_{+}}$. Assume that $w_{2} \in Y$ and let

$$
\left\|W_{1}\right\|_{Y} \vee\|W\|_{Y} \leq a<\infty
$$

Let $B_{0}$ satisfy the estimate (2.12) for $0 \leq m \leq k$. Then the following estimates hold for all $t \geq 1$ :

$$
\begin{gathered}
\left\|R_{1 \nu}(W, S)\right\|_{2} \leq C\left(a_{+}\right) t^{-2} \ell n t\|\nabla h\|_{2}+C a_{+}\left\{b_{0} t^{-1}\|h\|_{2}\right. \\
\left.+\left\|\partial_{t} h\right\|_{2}+a^{2} t^{-1}\left(\|h\|_{2}+I_{0}\left(\|h\|_{2}\right)\right)\right\}, \quad(3 . \\
\left\|\omega^{k} R_{1 \nu}(W, S)\right\|_{2} \leq C\left(a_{+}\right) t^{-2} \ell n t\left(\left\|\omega^{k} \nabla h\right\|_{2}+\left\|\omega^{\delta} \nabla h\right\|_{2}\right) \\
+C a_{+}\left\{b_{0}\left(t^{k-1-\delta / 3}\left\|\omega^{\delta} h\right\|_{2}+t^{-1}\left\|\omega^{k} h\right\|_{2}\right)+\left\|\omega^{k} \partial_{t} h\right\|_{2}+\left\|\omega^{\delta} \partial_{t} h\right\|_{2}\right. \\
\left.+t^{-2}\left(\left\|\omega^{k+1} h\right\|_{2}+\left\|\omega^{2} h\right\|_{2}\right)+t^{-1} a^{2}\left(\left\|\omega^{k} h\right\|_{2}+I_{k-1}\left(\left\|\omega^{k-1} h\right\|_{2}\right)\right)\right\}
\end{gathered}
$$

for $0<\delta<1 / 2$,

$$
\left\|\omega^{m} R_{2 \nu}(W, S)\right\|_{2} \leq t^{-1}\left\|\omega^{m+1} B_{0 L}\right\|_{2}+C a a_{+} t^{-1+\beta m} I_{0}\left(\|h\|_{2}\right)
$$


for all $m \geq 0$.

Proof. We first consider $\left\|R_{1 \nu}(W, S)\right\|_{2}$. We estimate successively

$$
\begin{aligned}
\left\|Q\left(S, w_{2}\right)\right\|_{2} \leq & \|S\|_{\infty}\left(\left\|w_{0}\right\|_{\infty}\|\nabla h\|_{2}+\left\|\nabla w_{0}\right\|_{3}\|h\|_{6}\right) \\
& +\|\nabla S\|_{3}\left\|w_{0}\right\|_{\infty}\|h\|_{6} \leq C\left(a_{+}\right) \ell n t\|\nabla h\|_{2}
\end{aligned}
$$

by (3.37) (3.38) (3.40) and Lemma 2.1,

$$
\left\|B_{0 S} w_{2}\right\|_{2} \leq\left\|B_{0 S}\right\|_{\infty}\left\|w_{0}\right\|_{\infty}\|h\|_{2} \leq C b_{0} a_{+}\|h\|_{2},
$$

by (2.14) and (3.37),

$$
\begin{gathered}
\left\|\left(\partial_{t} h\right) w_{0}\right\|_{2} \leq\left\|w_{0}\right\|_{\infty}\left\|\partial_{t} h\right\|_{2} \leq C a_{+}\left\|\partial_{t} h\right\|_{2}, \\
\left\|\nabla h \cdot \nabla w_{0}\right\|_{2} \leq\left\|\nabla w_{0}\right\|_{\infty}\|\nabla h\|_{2} \leq C a_{+}\|\nabla h\|_{2}, \\
\left\|B_{S}(W, W) w_{2}\right\|_{2} \leq\left\|B_{S}(W, W)\right\|_{\infty}\left\|w_{0}\right\|_{\infty}\|h\|_{2} \leq C a_{+} a^{2}\|h\|_{2},
\end{gathered}
$$

by estimating $B_{S}(W, W)$ in a way similar to that in Lemma 3.1,

$$
\begin{aligned}
& \left\|B_{S}\left(W+W_{1}, w_{2}\right) W_{1}\right\|_{2} \leq C\left\|W_{1}\right\|_{3}\left\|\omega B_{1}\left(W+W_{1}, w_{2}\right)\right\|_{2} \\
& \leq C\left\|W_{1}\right\|_{3}\left\|W+W_{1}\right\|_{\infty}\left\|w_{0}\right\|_{\infty} I_{0}\left(\|h\|_{2}\right) \leq C a_{+} a^{2} I_{0}\left(\|h\|_{2}\right)(3.54)
\end{aligned}
$$

by Lemma 2.1 and by (2.8). Collecting (3.49)-(3.54) yields (3.46).

We next consider $\omega^{k} R_{1 \nu}(W, S)$. We estimate successively

$$
\begin{aligned}
& \left\|\omega^{k} Q\left(S, w_{2}\right)\right\|_{2} \leq C\left\{\left\|w_{0}\right\|_{*}\|S\|_{*}\left\|\omega^{k+1} h\right\|_{2}+\left(\left\|w_{0}\right\|_{*}\left\|\omega^{k+1} S\right\|_{3 /(1+\delta)}\right.\right. \\
& \left.\left.+\left\|\omega^{k+1} w_{0}\right\|_{3 /(1+\delta)}\|S\|_{*}\right)\left\|\omega^{\delta} \nabla h\right\|_{2}\right\} \\
& \leq C\left(a_{+}\right) \ln t\left(\left\|\omega^{k} \nabla h\right\|_{2}+\left\|\omega^{\delta} \nabla h\right\|_{2}\right)
\end{aligned}
$$

by (3.37) (3.38) (3.40) and Lemmas 2.1 and 2.2, with

$$
\begin{aligned}
& \|f\|_{*}=\|f\|_{\infty}+\|\nabla f\|_{3}, \\
& \left\|\omega^{k}\left(B_{0 S} w_{2}\right)\right\|_{2} \leq C\left\{\left\|B_{0}\right\|_{\infty}\left\|w_{0}\right\|_{\infty}\left\|\omega^{k} h\right\|_{2}+\left(\left\|\omega^{k} B_{0}\right\|_{3 / \delta}\left\|w_{0}\right\|_{\infty}\right.\right. \\
& \left.\left.+\left\|B_{0}\right\|_{\infty}\left\|\omega^{k} w_{0}\right\|_{3 / \delta}\right)\left\|\omega^{\delta} h\right\|_{2}\right\} \\
& \leq C b_{0} a_{+}\left(\left\|\omega^{k} h\right\|_{2}+t^{k-\delta / 3}\left\|\omega^{\delta} h\right\|_{2}\right)
\end{aligned}
$$


by Lemmas 2.1 and 2.2 and by (2.14) (3.37),

$$
\begin{aligned}
\left\|\omega^{k}\left(\left(\partial_{t} h\right) w_{0}\right)\right\|_{2} & \leq C\left(\left\|w_{0}\right\|_{\infty}\left\|\omega^{k} \partial_{t} h\right\|_{2}+\left\|\omega^{k} w_{0}\right\|_{3 / \delta}\left\|\omega^{\delta} \partial_{t} h\right\|_{2}\right) \\
& \leq C a_{+}\left(\left\|\omega^{k} \partial_{t} h\right\|_{2}+\left\|\omega^{\delta} \partial_{t} h\right\|_{2}\right)
\end{aligned}
$$

by Lemmas 2.1 and 2.2 and by (3.37),

$$
\begin{aligned}
\left\|\omega^{k}\left(\nabla h \cdot \nabla w_{0}\right)\right\|_{2} & \leq C\left(\left\|\nabla w_{0}\right\|_{\infty}\left\|\omega^{k+1} h\right\|_{2}+\left\|\omega^{k+1} w_{0}\right\|_{3}\|\nabla h\|_{6}\right) \\
& \leq C a_{+}\left(\left\|\omega^{k+1} h\right\|_{2}+\left\|\omega^{2} h\right\|_{2}\right)
\end{aligned}
$$

by Lemmas 2.1 and 2.2 and by (3.37). We next estimate

$$
\begin{aligned}
& \left\|\omega^{k}\left(B_{S}(W, W) w_{2}\right)\right\|_{2} \leq C\left(\left\|B_{S} w_{0}\right\|_{\infty}+\left\|\omega^{k}\left(B_{S} w_{0}\right)\right\|_{3 / k}\right)\left\|\omega^{k} h\right\|_{2} \\
& \leq C\left(\left\|B_{S}\right\|_{\infty}+\left\|\omega^{k} B_{S}\right\|_{3 / k}\right)\left(\left\|w_{0}\right\|_{\infty}+\left\|\omega^{k} w_{0}\right\|_{3 / k}\right)\left\|\omega^{k} h\right\|_{2} \\
& \leq C a_{+} a^{2}\left\|\omega^{k} h\right\|_{2}
\end{aligned}
$$

where we have omitted the arguments in $B_{S}$, by Lemmas 2.1 and 2.2 and by (3.37) (3.45), and after estimating $B_{S}$ in a way similar to that in Lemma 3.1 . In the same way

$$
\begin{aligned}
& \left\|\omega^{k}\left(B_{S}\left(W+W_{1}, w_{2}\right) W_{1}\right)\right\|_{2} \leq C\left(\left\|W_{1}\right\|_{\infty}+\left\|\omega^{k} W_{1}\right\|_{3 / k}\right)\left\|\omega^{k} B_{1}\left(W+W_{1}, w_{2}\right)\right\|_{2} \\
& \leq C a I_{k-1}\left(\left\|\omega^{k-1} h\right\|_{2}\left(\left\|w_{0}\left(W+W_{1}\right)\right\|_{\infty}+\left\|\omega^{k-1}\left(W+W_{1}\right) w_{0}\right\|_{3 /(k-1)}\right)\right) \\
& \leq C a_{+} a^{2} I_{k-1}\left(\left\|\omega^{k-1} h\right\|_{2}\right) .
\end{aligned}
$$

Collecting (3.55)-(3.60) yields (3.47).

We finally estimate $R_{2 \nu}$. From (2.6) (2.8) we obtain

$$
\begin{gathered}
\left\|\omega^{m} R_{2 \nu}(W, S)\right\|_{2} \leq t^{-1}\left\|\omega^{m+1} B_{0 L}\right\|_{2} \\
+C t^{-1+\beta m} I_{0}\left(\left\|W+W_{1}\right\|_{\infty}\left\|w_{0}\right\|_{\infty}\|h\|_{2}\right)
\end{gathered}
$$

which yields (3.48) by using (3.37) (3.45).

In order to complete the estimate of the parts $R_{i \nu}(W, S), i=1,2$, of the remainders, we now estimate $h$ and $B_{0 L}$. Those estimates require some restrictions on the behaviour of $\left(F A_{+}, F \dot{A}_{+}\right)$at $\xi=0$. Those restrictions are imposed in a dilation homogeneous way through the use of a parameter $\mu \in(-1,1)$ in terms of quantities which have the same scaling properties as $\left\|A_{+} ; \dot{H}^{-3 / 2-\mu}\right\|$ and $\left\|\dot{A}_{+} ; \dot{H}^{-5 / 2-\mu}\right\|$. 
They will be further discussed at the end of this section.

Lemma 3.4. Let $1<k<3 / 2$ and $-1<\mu<1$. Let $\left(A_{+}, \dot{A}_{+}\right)$satisfy the conditions

$$
\begin{gathered}
A_{+} \in H^{k-1} \quad, \quad \dot{A}_{+} \in L^{2}, \\
x A_{+} \in H^{k-1} \quad, \quad x \dot{A}_{+} \in L^{3 / 2}, \\
x A_{+} \in \dot{H}^{-1 / 2-\mu}, \quad A_{+}, x \dot{A}_{+} \in \dot{H}^{-3 / 2-\mu}, \quad \dot{A}_{+} \in \dot{H}^{-5 / 2-\mu} .
\end{gathered}
$$

Let $B_{0 L}$ and $h$ be defined by (1.17) and (3.31).

Then the following estimates hold:

$$
\left\|\omega^{m} B_{0 L}\right\|_{2} \leq C t^{m-1 / 2+\left(\beta_{0}-1\right)(m+3 / 2+\mu)}\left(\left\|A_{+} ; \dot{H}^{-3 / 2-\mu}\right\|+\left\|\dot{A}_{+} ; \dot{H}^{-5 / 2-\mu}\right\|\right)
$$

for all $m \geq 0$,

$$
\left\|\omega^{m} h\right\|_{2} \leq 2 t^{m-3 / 2}\left(1 \vee t^{\left(\beta_{0}-1\right)(m-1 / 2+\mu)}\right)\left(\left\|A_{+} ; \dot{H}^{\rho}\right\|+\left\|\dot{A}_{+} ; \dot{H}^{\rho-1}\right\|\right)
$$

for all $m \leq k+1$, where

$$
\begin{gathered}
\rho=(m-2) \vee(-3 / 2-\mu)=-3 / 2-\mu+(m-1 / 2+\mu) \vee 0, \\
\left\|\omega^{m} \partial_{t} h\right\|_{2} \leq C t^{m-5 / 2}\left(1 \vee t^{\left(\beta_{0}-1\right)(m-1 / 2+\mu)}\right)\left(\left\|x A_{+} ; \dot{H}^{\rho+1}\right\|\right. \\
\left.\quad+\left\|x \dot{A}_{+} ; \dot{H}^{\rho}\right\|+\left\|A_{+} ; \dot{H}^{\rho}\right\|+\left\|\dot{A}_{+} ; \dot{H}^{\rho-1}\right\|\right)
\end{gathered}
$$

for all $m \leq k$ and $\rho$ given by (3.66),

$$
\|h\|_{\infty} \leq C\left(A_{+}, \dot{A}_{+}\right)
$$

where the constant depends on $\left(A_{+}, \dot{A}_{+}\right)$through the norms in (3.61) (3.63) ${ }_{\mu}$.

Proof. (3.64) follows immediately from the definitions (1.4) (1.13) and (1.17) of $A_{0}$ and $B_{0 L}$, from $(2.6)$ and from $(3.63)_{\mu}$.

In order to derive the estimates of $h$, it is convenient to come back to the variable $A_{0}$. The definition (3.31) of $h$ can be rewritten as

$$
h=2 t^{2} \omega^{-2} D_{0}^{-1} A_{0 S}=D_{0}^{-1} f
$$

where

$$
f=2 \omega^{-2} A_{0 S},
$$


$A_{0 S}$ is defined by

$$
A_{0 S}=t^{-1} D_{0}^{-1} B_{0 S}=\chi_{S} A_{0} \equiv F^{-1}\left(1-\chi\left(\xi t^{1-\beta_{0}}\right)\right) F A_{0}
$$

and $\chi$ is defined before (1.16).

(3.65). We estimate

$$
\begin{gathered}
\left\|\omega^{m} h\right\|_{2}=t^{m-3 / 2}\left\|\omega^{m} f\right\|_{2}=2 t^{m-3 / 2}\left\|\omega^{m-2} A_{0 S}\right\|_{2} \\
\leq 2 t^{m-3 / 2}\left(1 \vee t^{\left(\beta_{0}-1\right)(m-1 / 2+\mu)}\right)\left\|\omega^{\rho} A_{0}\right\|_{2}
\end{gathered}
$$

and the result follows from the assumptions (3.61) $(3.63)_{\mu}$.

(3.66). We use in addition the commutation relations

$$
t \partial_{t}=D_{0}^{-1} P D_{0} \quad, \quad P \omega^{-j}=\omega^{-j}(P+j) \quad, \quad\left[P, e^{i \omega t}\right]=0
$$

where $P$ is the dilation generator

$$
P=t \partial_{t}+x \cdot \nabla
$$

In particular

$$
\partial_{t} h=t^{-1} t \partial_{t} D_{0}^{-1} f=t^{-1} D_{0}^{-1} P f .
$$

Using the commutation relations (3.73), we compute

$$
\begin{aligned}
(1 / 2) P f & =\omega^{-2}(P+2) A_{0 S} \\
& =\omega^{-2} \cos \omega t(P+2) \chi_{S} A_{+}+\omega^{-3} \sin \omega t(P+3) \chi_{S} \dot{A}_{+} .
\end{aligned}
$$

Using the fact that $P+3=t \partial_{t}+\nabla \cdot x$ and the commutation relation

$$
\left[P, \chi_{S}\right]=-\beta F^{-1} \xi t^{1-\beta} \cdot \nabla \chi\left(\xi t^{1-\beta}\right) F \equiv \tilde{\chi}
$$

we obtain

$$
\begin{aligned}
(1 / 2) P f & =\omega^{-2} \cos \omega t \nabla \cdot \chi_{S} x A_{+}+\omega^{-3} \sin \omega t \nabla \cdot \chi_{S} x \dot{A}_{+} \\
& +\omega^{-2} \cos \omega t\left(\tilde{\chi}-\chi_{S}\right) A_{+}+\omega^{-3} \sin \omega t \tilde{\chi} \dot{A}_{+} .
\end{aligned}
$$

We then estimate

$$
\left\|\omega^{m} \partial_{t} h\right\|_{2}=t^{m-5 / 2}\left\|\omega^{m} \operatorname{Pf}\right\|_{2}
$$

and we estimate the contribution of the various terms of (3.75) exactly as in the proof of $(3.65)$, with $\left(m, A_{+}, \dot{A}_{+}\right)$replaced by $\left(m-1, x A_{+}, x \dot{A}_{+}\right)$in the first two 
terms, and with $\chi_{S}$ replaced by $\tilde{\chi}-\chi_{S}$ or by $\tilde{\chi}$ in the last two terms. This yields (3.67).

(3.68). By Lemma 2.1,

$$
\|h\|_{\infty} \leq C\left\|\omega^{3 / 2-\varepsilon} h\right\|_{2}^{1 / 2}\left\|\omega^{3 / 2+\varepsilon} h\right\|_{2}^{1 / 2}
$$

and (3.68) follows from (3.65) with $0<\varepsilon \leq(k-1 / 2) \wedge(\mu+1)$.

We now collect the results of Lemmas 3.2, 3.3 and 3.4 in order to exhibit a set of assumptions which imply those of Proposition 3.1

Proposition 3.2. Let $1<k<3 / 2<\ell$. Let $\mu, \lambda_{0}, \lambda, \beta_{0}, \beta$ and $k_{+}$satisfy the conditions

$$
\begin{gathered}
-1 / 4<\mu \leq 1 / 2 \\
\lambda>0 \quad, \quad(1<) \lambda+k<\lambda_{0}<7 / 6+2 \mu / 3(\leq 3 / 2) \\
0<\beta_{0} \leq \beta<2 / 3 \quad, \quad \beta(\ell+1)<\lambda_{0} \\
\beta_{0}(1 / 2-\mu)>\lambda_{0}-1-\mu \\
\beta_{0}(\mu+5 / 2)<2+\mu-\lambda_{0} \\
k_{+} \geq k+2 \quad, \quad \beta\left(k_{+}+1\right) \geq \lambda_{0} \quad, \quad \beta\left(\ell+3-k_{+}\right)<1 .
\end{gathered}
$$

Let $w_{+} \in H^{k_{+}}$and let $\left(A_{+}, \dot{A}_{+}\right)$satisfy (2.10) (3.62) (3.63) $)_{\mu}$. Let $(W, S)$ be defined by (3.25)-(3.29) (3.31).

Then $(W, S)$ satisfy the estimates (3.17) (3.18) (3.19) (3.20) (3.21) (with $0<$ $\eta<1-3 \beta / 2$ in (3.18)) and all the assumptions of Proposition 3.1 are satisfied.

Proof. The contribution of the terms not containing $w_{2}$ or $B_{0 L}$ in $(W, S)$ and in the remainders are estimated by Lemma 3.2 in the same way as in I. We concentrate on the remaining terms. The terms containing $w_{2}$ are estimated by Lemma 3.3 in terms of $h$, and $h$ and $B_{0 L}$ are estimated by Lemma 3.4.

The condition (3.17) restricted to $w_{2}=h w_{0}$ follows from the fact that it holds for $h$ by (3.65) (3.68) and trivially for $w_{0}$, and that it is multiplicative. Together with (3.45) for $W_{1}$, it implies (3.45) for $W$.

We next consider $R_{1 \nu}(W, S)$. Its $L^{2}$ norm is estimated by (3.46). By Lemma 3.4, it satisfies the estimate (3.19) provided

$$
\left(1-\beta_{0}\right)((1 / 2-\mu) \vee 0)<3 / 2-\lambda_{0}
$$


which reduces to $(3.81)$ for $\mu \leq 1 / 2$.

Similarly, $R_{1 \nu}(W, S)$ is estimated in $\dot{H}^{k}$ norm by (3.47) and satisfies the estimate (3.20) for $\delta$ sufficiently small under the condition (3.84) because the time decay of (3.47) is worse than that of (3.46) at worst by a factor $t^{k+2 \delta / 3}$ which is better than the allowed $t^{\lambda_{0}-\lambda}$ for $0<2 \delta / 3 \leq \lambda_{0}-\lambda-k$.

We now turn to $R_{2 \nu}(W, s)$. The contribution of $B_{0 L}$ is estimated by (3.64) and satisfies the estimate (3.21) provided

$$
m+1 / 2+\left(\beta_{0}-1\right)(m+7 / 2+\mu) \leq-1-\lambda_{0}+\beta(m+1)
$$

which is implied by (3.82) for $\beta_{0} \leq \beta$.

The term containing $w_{2}$ is estimated by (3.48) and satisfies the estimate (3.21) by (3.65) under the condition (3.84).

We remark here that the upper bound on $\lambda_{0}$ in (3.79) is the compatibility condition of (3.81) (3.82). The remaining conditions in (3.78)-(3.83) come from I.

We now comment briefly on the various parameters that occur in Proposition 3.2 and on the conditions (3.78)-(3.83) that they have to satisfy. The parameters $k$ and $\ell$ characterize the regularity of the spaces of resolution for $(w, s)$. As a consequence, $k$ also characterizes the regularity of $\left(A_{+}, \dot{A}_{+}\right)$as given by $(2.10)$. The parameter $\mu$ characterizes the behaviour of $\left(\widehat{A}_{+}, \widehat{\dot{A}}_{+}\right)=\left(F A_{+}, F \dot{A}_{+}\right)$at $\xi=0$ through the condition $(3.63)_{\mu}$. The parameters $\lambda_{0}$ and $\lambda$ are the time decay exponents of the norms of $q$ in $L^{2}$ and in $\dot{H}^{k}$. The $\mu$ dependent upper bound on $\lambda_{0}$ in (3.79) ranges over $(1,3 / 2]$ when $\mu$ ranges over $(-1 / 4,1 / 2]$. Since the condition (3.84) saturates at $\lambda_{0}<3 / 2$ for $\mu \geq 1 / 2$, there is no point in considering values of $\mu>1 / 2$. The parameters $\beta_{0}$ and $\beta$ characterize the splitting of $B_{0}$ and $B_{1}$ respectively into short range and long range parts, and therefore the splitting of the Schrödinger equation into transport and Hamilton-Jacobi equations. The parameter $\beta$ should not be too large and can be taken equal to $\beta_{0}$. The parameter $\beta_{0}$ satisfies two inequalities (3.81) and (3.82) in opposite directions, depending on $\lambda_{0}$ and $\mu$, and expressing the fact that $B_{0 S}$ and $B_{0 L}$ are not too large. The upper bound on $\lambda_{0}$ in (3.79) is the compatibility condition of those inequalities. Whenever it is satisfied, the value $\beta_{0}=1 / 3$ is allowed. Actually both (3.81) and (3.82) reduce to that upper bound for $\beta_{0}=1 / 3$. Finally $k_{+}$characterizes the regularity of $w_{+}$and should be sufficiently large, depending on $k, \ell, \lambda_{0}$ and $\beta$. 
Remark 3.1. For $\mu=1 / 2$ the short range restriction is no longer needed in the estimates of $h$ and $\partial_{t} h$ in Lemma 3.4, and therefore the splitting of $B_{0}$ into short range and long range parts is no longer needed, namely $B_{0}$ can be kept entirely in the equation for $w$.

We finally discuss the condition (3.63) $)_{\mu}$ of Lemma 3.4. That condition restricts the behaviour of the relevant functions for small $|\xi|$ in Fourier transformed variables. Let $A$ be any of the functions $A_{+}, \dot{A}_{+}, x A_{+}, x \dot{A}_{+}$and define $A_{<}$by $\widehat{A}_{<}(\xi)=$ $\chi(\xi) \widehat{A}(\xi)$. Then the conditions on $A_{<}$contained in $(3.63)_{\mu}$ all take the form

$$
A_{<} \in \dot{H}^{-3 / 2-\nu}
$$

for $\nu=\mu, \mu \pm 1$. We first remark that in the proof of Lemma 3.4, all such conditions can be replaced by

$$
|\xi|^{-\nu} \widehat{A}_{<} \in L^{\infty}
$$

at the expense of inserting an additional factor $(\ln t)^{1 / 2}$ in (3.65) in the case of equality, namely for $m=1 / 2-\mu$. This follows from the fact that

$$
\left\||\xi|^{m-3 / 2} \widehat{A}_{S<}\right\|_{2} \leq \begin{cases}C\left\||\xi|^{-\nu} \widehat{A}_{<}\right\|_{\infty}\left(1 \vee t^{\left(\beta_{0}-1\right)(m+\nu)}\right) & \text { for } m \neq-\nu \\ C\left\||\xi|^{-\nu} \widehat{A}_{<}\right\|_{\infty}(\ell n t)^{1 / 2} & \text { for } m=-\nu\end{cases}
$$

The occurrence of the factor $(\ell n t)^{1 / 2}$ is harmless for the applications. The condition (3.86) is weaker than (3.87) as regards the behaviour of $\hat{A}_{<}$away from zero, since it requires only $\widehat{A}_{<} \in L_{\text {loc }}^{2}$ instead of $\widehat{A}_{<} \in L_{\text {loc }}^{\infty}$. Furthermore (3.86) almost follows from (3.87), up to a change of $\nu$ into $\nu+\varepsilon$. In fact

$$
\left\||\xi|^{-3 / 2-\nu} \hat{A}_{<}\right\|_{2} \leq C \varepsilon^{-1 / 2}\left\||\xi|^{-\nu-\varepsilon} \hat{A}_{<}\right\|_{\infty}
$$

for $\varepsilon>0$. In addition, under the short range condition

$$
\left\||\xi|^{-3 / 2-\nu} \widehat{A}_{S<}\right\|_{2} \leq C(\ln t)^{1 / 2}\left\||\xi|^{-\nu} \widehat{A}_{<}\right\|_{\infty}
$$

which is the special case $m=-\nu$ of (3.88).

The restrictions on $\left(\widehat{A}_{+}, \widehat{\dot{A}}_{+}\right)$at $\xi=0$ expressed by (3.86) have the unpleasant feature that for $\nu \geq 0$ they cannot be ensured by imposing decay of $\left(A_{+}, \dot{A}_{+}\right)$at infinity in space and that they require in addition some moment conditions. For instance even for $A \in \mathcal{S}$ one has

$$
\omega^{-3 / 2-\nu} A_{<}=C|x|^{-3 / 2+\nu} * A_{<}
$$


for $|\nu|<3 / 2[9]$, which behaves as

$$
|x|^{-3 / 2+\nu} \int A d x
$$

as $|x| \rightarrow \infty$ and therefore cannot be in $L^{2}$ for $\nu \geq 0$ unless $\int A d x=0$. More generally when $\nu$ increases, vanishing of the $n$-th moment of $A$ is necessary as soon as $\nu \geq n$. Actually the parameter $\mu$ in $(3.63)_{\mu}$ has been introduced in order to minimize the number of such conditions by taking $\mu$ small.

We now give sufficient conditions on $\left(A_{+}, \dot{A}_{+}\right)$in terms of space decay and vanishing of suitable moments so as to ensure the low frequency part of $(3.63)_{\mu}$.

Lemma 3.5. Let $-1<\mu<1$. Let $\left(A_{+}, \dot{A}_{+}\right)$satisfy (3.61) (3.62) and in addition

$$
\begin{gathered}
x A_{+} \in L^{3 /(2+\mu) \vee 2}, \int \dot{A}_{+} d x=0,<x>^{1+\mu+\varepsilon} \quad \dot{A}_{+} \in L^{1}, \\
A_{+}, x \dot{A}_{+} \in L^{3 /(3+\mu)} \quad \text { for } \mu<0, \\
\int A_{+} d x=\int x \dot{A}_{+} d x=0 \quad, \quad<x>^{\mu+\varepsilon} \quad A_{+} \in L^{1} \quad \text { for } \mu \geq 0 .
\end{gathered}
$$

Then $(3.63)_{\mu}$ holds.

Proof. The high frequency part of $\left(A_{+}, \dot{A}_{+}\right)$is controlled by (3.61) (3.62) and it is sufficient to consider $\left(A_{+<}, \dot{A}_{+<}\right)$, although in some cases the high frequency parts are also controlled by (3.91) (3.92).

We first consider $x A_{+}$. For $-1 / 2 \leq \mu<1$, we estimate

$$
\left\|\omega^{-1 / 2-\mu} x A_{+}\right\|_{2} \leq C\left\|x A_{+}\right\|_{3 /(2+\mu)}
$$

by Lemma 2.1. For $\mu \leq-1 / 2$, we estimate simply

$$
\left\|\omega^{-1 / 2-\mu} x A_{+<}\right\|_{2} \leq C\left\|x A_{+}\right\|_{2}
$$

We next consider $A_{+}$and $x \dot{A}_{+}$together and we use $A$ to denote either of them. For $-1<\mu<0$, we estimate

$$
\left\|\omega^{-3 / 2-\mu} A\right\|_{2} \leq C\|A\|_{3 /(3+\mu)}
$$

by Lemma 2.1 . For $\mu \geq 0$, we estimate

$$
|\xi|^{-\mu-\varepsilon}|\widehat{A}(\xi)|=(2 \pi)^{-3 / 2}|\xi|^{-\mu-\varepsilon}\left|\int d x(\exp (-i x \xi)-1) A(x)\right|
$$




$$
\leq C\left\||x|^{\mu+\varepsilon} A\right\|_{1}
$$

for $0 \leq \mu+\varepsilon \leq 1$. The required estimate then follows from (3.61) (3.62) (3.91) (3.93).

We finally consider $\dot{A}_{+}$. For $\mu<0$, we apply the previous result with $A$ replaced by $\dot{A}_{+}$and $\mu$ replaced by $\mu-1$. For $\mu \geq 0$, we estimate

$$
\begin{gathered}
|\xi|^{-1-\mu-\varepsilon}|\widehat{\dot{A}}(\xi)|=(2 \pi)^{-3 / 2}|\xi|^{-1-\mu-\varepsilon} \int d x(\exp (-i x \xi)-1-i x \xi) \dot{A}_{+}(x) \\
\leq C\left\||x|^{1+\mu+\varepsilon} \dot{A}_{+}\right\|_{1}
\end{gathered}
$$

for $0 \leq \mu+\varepsilon \leq 1$. The required estimate then follows from (3.61) (3.91) (3.93).

\section{Wave operators and asymptotics for ( $u, A)$}

In this section we complete the construction of the wave operators for the system (1.1) (1.2) and we derive asymptotic properties of solutions in their range. The construction relies in an essential way on Propositions 3.1 and 3.2. So far we have worked with the system (1.20) for $(w, s)$ and the first task is to reconstruct the phase $\varphi$. Corresponding to $S=s_{0}+s_{1}$, we define $\phi=\varphi_{0}+\varphi_{1}$ where

$$
\begin{gathered}
\varphi_{0}=-\int_{1}^{t} d t^{\prime} t^{\prime-1} B_{L}\left(w_{0}\left(t^{\prime}\right), w_{0}\left(t^{\prime}\right)\right), \\
\varphi_{1}=-\int_{t}^{\infty} d t^{\prime}\left(2 t^{\prime 2}\right)^{-1}\left|s_{0}\left(t^{\prime}\right)\right|^{2}+2 \int_{t}^{\infty} d t^{\prime} t^{\prime-1} B_{L}\left(w_{0}\left(t^{\prime}\right), w_{1}\left(t^{\prime}\right)\right),
\end{gathered}
$$

so that $s_{0}=\nabla \varphi_{0}$ and $s_{1}=\nabla \varphi_{1}$.

Let now $(w, s)$ be the solution of the system (1.20) constructed in Proposition 3.1 and let $(q, \sigma)=(w, s)-(W, S)$. We define

$$
\begin{aligned}
& \psi=-\int_{t}^{\infty} d t^{\prime}\left(2 t^{\prime 2}\right)^{-1}\left(\sigma \cdot(\sigma+2 S)+s_{1} \cdot\left(s_{1}+2 s_{0}\right)\right)\left(t^{\prime}\right) \\
& +\int_{t}^{\infty} d t^{\prime} t^{\prime-1}\left(B_{L}(q, q)+2 B_{L}(W, q)+B_{L}\left(w_{1}, w_{1}\right)\right)\left(t^{\prime}\right)
\end{aligned}
$$

which is taylored to ensure that $\nabla \psi=\sigma$, given the fact that $s_{0}, s_{1}$ and $\sigma$ are gradients. The integral is easily seen to converge in $\dot{H}^{1}$ (see I.8.4), and to satisfy

$$
\|\nabla \psi\|_{2}=\|\sigma\|_{2} \leq C t^{-\lambda_{0}}
$$


Finally we define $\varphi=\phi+\psi$ so that $\nabla \varphi=s$, and $(w, \varphi)$ solves the system (1.18). For more details on the reconstruction of $\varphi$ from $s$, we refer to Section 8 of I.

We can now define the wave operators for the system (1.1) (1.2) as follows. We start from the asymptotic state $\left(u_{+}, A_{+}, \dot{A}_{+}\right)$for $(u, A)$. We define $w_{+}=F u_{+}$, we define $B_{0}$ by (1.4) (1.13), namely

$$
A_{0}=\dot{K}(t) A_{+}+K(t) \dot{A}_{+}=t^{-1} D_{0} B_{0}
$$

and we define $(W, S)$ by (3.25)-(3.29) (3.31).

We next solve the system (1.20) with infinite initial time by Propositions 3.1 and 3.2 and we reconstruct $\varphi$ from $s$ as explained above, namely $\varphi=\varphi_{0}+\varphi_{1}+\psi$ with $\varphi_{0}, \varphi_{1}$ and $\psi$ defined by (4.1) (4.2) (4.3) with $(q, \sigma)=(w, s)-(W, S)$. We finally substitute $(w, \varphi)$ thereby obtained into (1.11) (1.3) thereby obtaining a solution $(u, A)$ of the system (1.1) (1.2). The wave operator is defined as the map $\Omega$ : $\left(u_{+}, A_{+}, \dot{A}_{+}\right) \rightarrow(u, A)$.

In order to state the regularity properties of $u$ that follow in a natural way from the previous construction, we introduce appropriate function spaces. In addition to the operators $M=M(t)$ and $D=D(t)$ defined by (1.8) (1.9), we introduce the operator

$$
J=J(t)=x+i t \nabla
$$

the generator of Galilei transformations. The operators $M, D, J$ satisfy the commutation relation

$$
i M D \nabla=J M D \text {. }
$$

For any interval $I \subset[1, \infty)$ and any $k \geq 0$, we define the space

$$
\begin{aligned}
\mathcal{X}^{k}(I) & =\left\{u: D^{*} M^{*} u \in \mathcal{C}\left(I, H^{k}\right)\right\} \\
& =\left\{u:<J(t)>^{k} u \in \mathcal{C}\left(I, L^{2}\right)\right\}
\end{aligned}
$$

where $\langle\lambda\rangle=\left(1+\lambda^{2}\right)^{1 / 2}$ for any real number or self-adjoint operator $\lambda$ and where the second equality follows from (4.6).

We now collect the information obtained for the solutions of the system (1.1) (1.2) and state the main result of this paper as follows.

Proposition 4.1. Let $1<k<3 / 2<\ell$. Let $\mu, \lambda_{0}, \lambda, \beta_{0}, \beta$ and $k_{+}$satisfy the conditions (3.78)-(3.83). 
Let $u_{+} \in F H^{k_{+}}$, let $w_{+}=F u_{+}$and $a_{+}=\left|w_{+}\right|_{k_{+}}$. Let $\left(A_{+}, \dot{A}_{+}\right)$satisfy (2.10) (3.62) (3.63) $)_{\mu}$ Let $(W, S)$ be defined by (3.25)-(3.29) (3.31). Then

(1) There exists $T, 1 \leq T<\infty$ such that the auxiliary system (1.20) has a unique solution $(w, s) \in \mathcal{C}\left([T, \infty), X^{k, \ell}\right)$ satisfying

$$
\begin{gathered}
\|w(t)-W(t)\|_{2} \leq C\left(a_{+}, A_{+}, \dot{A}_{+}\right) t^{-\lambda_{0}}, \\
\left\|\omega^{k}(w(t)-W(t))\right\|_{2} \leq C\left(a_{+}, A_{+}, \dot{A}_{+}\right) t^{-\lambda}, \\
\left\|\omega^{m}(s(t)-S(t))\right\|_{2} \leq C\left(a_{+}, A_{+}, \dot{A}_{+}\right) t^{-\lambda_{0}+\beta m} \text { for } 0 \leq m \leq \ell+1,
\end{gathered}
$$

for all $t \geq T$, where the constants $C\left(a_{+}, A_{+}, \dot{A}_{+}\right)$depend on $\left(A_{+}, \dot{A}_{+}\right)$through the norms associated with (2.10) (3.62) (3.63) $)_{\mu}$.

(2) Let $\phi=\varphi_{0}+\varphi_{1}$ be defined by (4.1) (4.2), let $\varphi=\phi+\psi$ with $\psi$ defined by (4.3) and $(q, \sigma)=(w, s)-(W, S)$. Let

$$
u=M D \exp (-i \varphi) w
$$

and define $A$ by (1.3) (1.4) (1.5). Then $u \in \mathcal{X}^{k}([T, \infty)),\left(A, \partial_{t} A\right) \in \mathcal{C}\left([T, \infty), H^{k} \oplus\right.$ $\left.H^{k-1}\right),(u, A)$ solves the system (1.1) (1.2) and $u$ behaves asymptotically in time as $M D \exp (-i \phi) W$ in the sense that it satisfies the following estimates :

$$
\begin{gathered}
\|u(t)-M(t) D(t) \exp (-i \phi(t)) W(t)\|_{2} \leq C\left(a_{+}, A_{+}, \dot{A}_{+}\right) t^{-\lambda_{0}} \\
\left\||J(t)|^{k}(\exp (i \phi(t, x / t)) u(t)-M(t) D(t) W(t))\right\|_{2} \leq C\left(a_{+}, A_{+}, \dot{A}_{+}\right) t^{-\lambda}, \\
\|u(t)-M(t) D(t) \exp (-i \phi(t)) W(t)\|_{r} \leq C\left(a_{+}, A_{+}, \dot{A}_{+}\right) t^{-\lambda_{0}+\left(\lambda_{0}-\lambda\right) \delta(r) / k}
\end{gathered}
$$

for $0 \leq \delta(r)=(3 / 2-3 / r) \leq k$, for all $t \geq T$.

Define in addition

$$
A_{2}=A-A_{0}-A_{1}\left(|D W|^{2}\right) .
$$

Then $A$ behaves asymptotically in time as $A_{0}+A_{1}\left(|D W|^{2}\right)$ in the sense that $A_{2}$ satisfies the following estimates:

$$
\begin{gathered}
\left\|A_{2}(t)\right\|_{2} \leq C\left(a_{+}, A_{+}, \dot{A}_{+}\right) t^{-\lambda_{0}+1 / 2} \\
\left\|\nabla A_{2}(t)\right\|_{2} \leq C\left(a_{+}, A_{+}, \dot{A}_{+}\right) t^{-2 \lambda_{0}-1 / 2+\left(\lambda_{0}-\lambda\right) 3 / 2 k} \\
\left\|\omega^{2 k-1 / 2} A_{2}(t)\right\|_{2} \leq C\left(a_{+}, A_{+}, \dot{A}_{+}\right) t^{-2 \lambda-2 k+1}
\end{gathered}
$$

for all $t \geq T$. 
(3) The solution $(u, A)$ also behaves asymptotically as $\left(M D \exp (-i \phi) W_{1}, A_{0}+\right.$ $A_{1}\left(\left|D W_{1}\right|\right)^{2}$ in the sense that the estimates (4.12)-(4.14) and (4.16)-(4.18) also hold with $W$ replaced by $W_{1}$ (see (3.25)).

Sketch of proof. Part (1) is a restatement of the conclusions of Proposition 3.1 supplemented by (4.4) and follows from Propositions 3.1 and 3.2.

Part (2) follows from Part (1) and is proved in exactly the same way as Part (2) of Proposition I.8.1.

Part (3) is proved in the same way as Part (2). It follows from the fact that the only estimates of $W$ and $q=w-W$ that are used in the proof of Part (2) are (3.45) which also holds for $W_{1}$ and (4.8) (4.9) which also hold for $w_{2}$. In fact, the latter estimates hold for $h$ by Lemma 3.4, especially (3.65), under the assumptions of Proposition 3.2 and follow therefrom for $w_{2}$ in a trivial way.

Remark 4.1. It may seem surprising that the improved asymptotic form $W$ for $w$ does not give rise to better asymptotic estimates than the simpler form $W_{1}$ in the norms (4.12)-(4.14) and (4.16)-(4.18). The reason is that the additional term $w_{2}$ is small and gives rise to small contributions in terms of those norms. This does not prevent that term to give a large contribution to the time derivative $\partial_{t} w$ in $(1.20)$ through the derivative term $t^{-2} \Delta w_{2}$. That contribution is essential to allow for the solution of the system (1.20) without assuming the support condition. The same phenomenon appears in [8].

Acknowledgements. We are grateful to Dr. A. Shimomura for enlightening discussions. 


\section{References}

[1] J. Ginibre, G. Velo : Long range scattering and modified wave operators for the Wave-Schrödinger system, Ann. H.P., 3, 537-612 (2002).

[2] L. Hörmander : The Analysis of Linear Partial Differential Operators, Vol I, Springer, Berlin, 1983.

[3] T. Kato, G. Ponce : Commutator estimates and the Euler and Navier-Stokes equations, Comm. Pure Appl. Math., 41, 891-907 (1988).

[4] C. Kenig, G. Ponce, L. Vega : The initial value problem for a class of nonlinear dispersive equations, in Functional-Analytic Methods for Partial Differential Equations, Lect. Notes Math., 1450, 141-156 (1990).

[5] T. Ozawa : Long range scattering for nonlinear Schrödinger equations in one space dimension, Commun. Math. Phys., 139, 479-493 (1991).

[6] T. Ozawa, Y. Tsutsumi : Asymptotic behaviour of solutions for the coupled Klein-Gordon-Schrödinger equations, in Spectral and Scattering Theory and Applications, Adv. Stud. in Pure Math., Jap. Math. Soc., 23, 295-305 (1994).

[7] A. Shimomura : Wave operators for the coupled Klein-Gordon-Schrödinger equations in two space dimensions, preprint (2002).

[8] A. Shimomura : Modified wave operators for the coupled Wave-Schrödinger equations in three space dimensions, preprint (2002).

[9] E. Stein : Singular integrals and differentiability properties of functions, Princeton Univ. Press, Princeton 1970. 